\title{
EXPONENTIAL RATE OF CONVERGENCE INDEPENDENT OF THE DIMENSION IN A MEAN-FIELD SYSTEM OF PARTICLES
}

\author{
BY
}

BARTŁOMIEJ DYDA (WROCŁAW) AND JULIAN TUGAUT (SAINT-ÉTIENNE AND Lyon)

\begin{abstract}
This article deals with a mean-field model. We consider a large number of particles interacting through their empirical law. We know that there is a unique invariant probability for this diffusion. We look at functional inequalities. In particular, we briefly show that the diffusion satisfies a Poincaré inequality. Then, we establish a so-called $W J$-inequality, which is independent of the number of particles.
\end{abstract}

2010 AMS Mathematics Subject Classification: Primary: 60F10; Secondary: 60J60, 60G10.

Key words and phrases: Mean-field model, Poincaré inequality, transportation inequality, high dimension.

\section{INTRODUCTION}

1.1. Model. First, we consider a sequence $\left(X_{0}^{i}\right)_{i \geqslant 1}$ of independent and identically distributed random variables with common law $\mu_{0}$ on $\mathbb{R}^{d}$. We also consider a sequence of independent Brownian motions $\left(B^{i}\right)_{i \geqslant 1}$ on $\mathbb{R}^{d}$. These Brownian motions are assumed to be independent of the previously introduced random variables.

The system of interacting particles that we look at evolves in the landscape of a potential $V$. This potential is denoted as the confining potential. Its effect is, roughly speaking, to locate the particles in a compact of $\mathbb{R}^{d}$. We assume that the confining potential $V$ is convex at infinity but not globally convex. However, we assume that the Hessian of $V$ is minorated:

$$
\nabla^{2} V \geqslant-\theta \mathrm{Id}
$$

where $\theta$ is a positive constant.

We now introduce the so-called interacting potential $F$. We do not assume that it is either convex or not convex. However, the following inequality is required:

$$
\nabla^{2} F \geqslant-\alpha \text { Id }
$$

The precise assumptions are given subsequently. We consider the following system 
of interacting particles for $1 \leqslant i \leqslant N$ :

$$
X_{t}^{i, N}=X_{0}^{i}+\sigma B_{t}^{i}-\int_{0}^{t} \nabla V\left(X_{s}^{i, N}\right) d s-\int_{0}^{t} \frac{1}{N} \sum_{j=1}^{N} \nabla F\left(X_{s}^{i, N}-X_{s}^{j, N}\right) d s .
$$

Here, $N$ is large.

This system of $N$ particles in $\mathbb{R}^{d}$ may be seen as one particle in $\mathbb{R}^{d N}$. Indeed, let us define

$$
\mathcal{X}_{t}^{N}:=\left(X_{t}^{1, N}, \ldots, X_{t}^{N, N}\right) \in \mathbb{R}^{d N} .
$$

Thus, the diffusion $\mathcal{X}$ is a simple diffusion evolving in the landscape of a potential of $\mathbb{R}^{d N}$,

$$
\mathcal{X}_{t}^{N}=\mathcal{X}_{0}^{N}+\sigma \mathcal{B}_{t}-N \int_{0}^{t} \nabla \Upsilon^{N}\left(\mathcal{X}_{s}^{N}\right) d s,
$$

where $\mathcal{B}:=\left(B^{1}, \ldots, B^{N}\right)$, and the potential $\Upsilon^{N}$ is defined as

$$
\Upsilon^{N}\left(X_{1}, \ldots, X_{N}\right):=\frac{1}{N} \sum_{i=1}^{N} V\left(X_{i}\right)+\frac{1}{2 N^{2}} \sum_{i=1}^{N} \sum_{j=1}^{N} F\left(X_{i}-X_{j}\right) .
$$

This model has a natural application in the financial markets in which there is a huge number of agents who act in function of the global behaviour of the system. We can also think about the system of exchanges between banks, a classical result being important for the interaction in the probability of bankruptcy.

With some other assumptions, these equations are used as a model for the growth of cerebral tumors. Indeed, each of the cells tries to be alone for developing itself.

Mean-field systems of particles are also used in social interactions (see [8]). They also appeared quite naturally in the study of stochastic partial differential equations, see [9].

\subsection{Well-known results.}

1.2.1. Existence. Basically, we make the following assumptions:

- The potential $V$ is convex at infinity. In fact, we even have $\nabla^{2} V(\infty)=\infty$.

- In the neighbourhood of infinity, the confining potential behaves as a polynomial function: $\langle\nabla V(x) ; x\rangle=\|x\|^{2 m}+o\left(\|x\|^{2 m}\right)$ at infinity, where $m \in \mathbb{N}$.

- In the neighbourhood of infinity, the interacting potential behaves as a polynomial function: $\langle\nabla F(x) ; x\rangle=\|x\|^{2 n}+o\left(\|x\|^{2 n}\right)$ at infinity, where $n \in \mathbb{N}$.

Then, we consider the maximal degree by putting $q:=\max \{m, n\}$. Thus, if the initial law admits a moment of order $2 q$, that is to say, if

$$
\int_{\mathbb{R}^{d}}\|x\|^{2 q} \mu_{0}(d x)<\infty,
$$

there exists a unique solution to equation (ㅍ.J). We denote by $\left(\mathcal{X}_{t}^{N}\right)_{t \geqslant 0}$ this diffusion solution. Let us remark that the previous assumption for the existence of a solution does not depend on the number $N$ of particles. 
1.2.2. Invariant probability. There is a unique invariant probability $\mu^{\sigma, N}$ on $\mathbb{R}^{d N}$ for diffusion (․ㅔ) defined by

$$
\mu^{\sigma, N}(d \mathcal{X}):=Z_{\sigma, N}^{-1} \exp \left\{-\frac{2 N}{\sigma^{2}} \Upsilon^{N}(\mathcal{X})\right\} d \mathcal{X}
$$

where the potential $\Upsilon^{N}$ is defined in (ㄴ.2).

This potential makes sense when $N$ goes to infinity. Indeed, it represents the energy associated with the probability $\frac{1}{N} \sum_{i=1}^{N} \delta_{X_{i}}$. We can observe that there is an $N$ in the factor so that the invariant probability and the long-time behaviour depend on $N$.

1.2.3. Long-time behaviour. Thanks to Bakry et al. [2], the measure $\mu^{\sigma, N}$ satisfies a Poincaré inequality

$$
\operatorname{Var}_{\mu^{\sigma, N}}(f) \leqslant \frac{1}{C_{\sigma}(N)} \int_{\mathbb{R}^{d N}}\|\nabla f\|^{2} d \mu^{\sigma, N}
$$

for any $f$ which is a smooth function from $\mathbb{R}^{d N}$ to $\mathbb{R}$. This inequality is equivalent to the convergence inequality

$$
\left\|\mathcal{P}_{t}^{N} f-\mathbb{E}_{\mu^{\sigma, N}}(f)\right\|_{L^{2}\left(\mu^{\sigma, N}\right)}^{2} \leqslant \exp \left\{-\frac{2}{C_{\sigma}(N)} t\right\} \operatorname{Var}_{\mu^{\sigma, N}}(f),
$$

where we have put $\mathcal{P}_{t}^{N} f(x):=\mathbb{E}_{x}\left\{f\left(\mathcal{X}_{t}^{N}\right)\right\}$.

Let us point out that the constant $C_{\sigma}(N)$ which appears in the inequality does not have any reason to be independent of the number of particles, that is, of the dimension of the space in which the solution $\mathcal{X}^{N}$ evolves.

1.2.4. Hydrodynamical limit. Intuitively, $\left(X_{t}^{i, N}\right)_{t \geqslant 0}$ behaves as the diffusion $\left(\bar{X}_{t}^{i}\right)_{t \geqslant 0}$ when $N$ is large. Here, the diffusion $\bar{X}^{i}$ is defined by the equation

$$
\bar{X}_{t}^{i}=X_{0}^{i}+\sigma B_{t}^{i}-\int_{0}^{t} \nabla V\left(\bar{X}_{s}^{i}\right) d s-\int_{0}^{t} \nabla F * \mu_{s}\left(\bar{X}_{s}^{i}\right) d s
$$

with $\mu_{s}:=\mathbb{P}_{\bar{X}_{s}^{1}}$. Indeed, we can observe that the influence of the particle $j$ on the particle 1 becomes small when $N$ is large. So, roughly speaking, the particles of the interacting system of particles become independent. However, equation (ㅍ.]) can be written in the form

$$
X_{t}^{i, N}=X_{0}^{i}+\sigma B_{t}^{i}-\int_{0}^{t} \nabla V\left(X_{s}^{i, N}\right) d s-\int_{0}^{t} \nabla F * \mu_{s}^{N}\left(X_{s}^{i, N}\right) d s,
$$

with $\mu_{s}^{N}:=\frac{1}{N} \sum_{j=1}^{N} \delta_{X_{s}^{j, N}}$. If the particles become independent, the measure $\mu_{s}^{N}$ converges to $\mu_{s}$, which explains why the particles $X^{i, N}$ intuitively are close to $\bar{X}^{i}$. 
We now assume $\mathbb{E}\left\{\left\|X_{0}^{1}\right\|^{8 q^{2}}\right\}<\infty$, that is,

$$
\int_{\mathbb{R}^{d}}\|x\|^{8 q^{2}} \mu_{0}(d x)<\infty,
$$

where we remind the reader that $q$ is the maximum of the degrees of $V$ and $F$. Under this assumption, we have a so-called propagation of chaos:

$$
\sup _{t \in[0, T]} \mathbb{E}\left\{\left\|X_{t}^{i, N}-\overline{X_{t}^{i}}\right\|^{2}\right\} \rightarrow 0
$$

for any $T>0$.

See [12], [14], [11], [10], [3], [4] for propagation of chaos.

1.3. Aim of the article. The following theorem is our main result.

THEOREM 1.1. Let the assumptions listed in Subsection $\square .5$ hold, and let $\sigma$ be large. Then the law of the diffusion $\mathcal{X}_{t}^{N}$ converges to the unique invariant probability $\mu^{\sigma, N}$ as $t \rightarrow \infty$ in the Wasserstein distance $\mathbb{W}_{2}$. Moreover, the rate of the convergence is exponential and uniform with respect to the number of particles.

1.4. Outline of the article. First, we present the assumptions of the article. In the next section, we justify why the diffusion satisfies a Poincare inequality. Then, we give some classical results on this inequality and discuss an eventual uniform Poincaré inequality. In Section 3, we present the framework of the paper. In Section 4, we give the main result and its proof. Finally, in the last section, we give the proof of a technical proposition which is of crucial interest in our work.

1.5. Assumptions. Let us present the assumptions of the paper.

(A1) $V$ is a smooth function on $\mathbb{R}^{d}$.

(A2) $V$ is convex at infinity: for any $\lambda>0$, there exists $R_{\lambda}>0$ such that $\nabla^{2} V(x)$ $>\lambda$ Id for any $\|x\| \geqslant R_{\lambda}$.

(A3) There exists a convex non-negative function $V_{0}$ such that $\nabla^{2} V_{0}(0)=0$ and $V(x)=V_{0}(x)-\frac{\theta}{2}\|x\|^{2}$ with $\theta>0$.

(A4) There exist $m \in \mathbb{N}^{*}$ and $C>0$ such that $\|\nabla V(x)\| \leqslant C\left(1+\|x\|^{2 m-1}\right.$ ) for all $x \in \mathbb{R}^{d}$.

(A5) The inequality $|V(x)| \leqslant c\|x\|^{2}$ holds for $\|x\| \leqslant 1$; in particular, $V(0)=0$.

(A6) $F(x)=G(\|x\|)-\frac{\alpha}{2}\|x\|^{2}$, where $G$ is a polynomial, even and convex function with degree equal to $\operatorname{deg}(G)=: 2 n \geqslant 2$ and $G(0)=0$. Here, $\alpha$ is not necessarily positive.

(A7) $\int_{\mathbb{R}^{d}}\|x\|^{8 q^{2}} \mu_{0}(d x)<\infty$ with $q:=\max \{m, n\}$.

(A8) The entropy of the probability measure is finite. In other words, $\mu_{0}$ is absolutely continuous with respect to the Lebesgue measure, and we have

$$
\int_{\mathbb{R}^{d}} u_{0}(x) \log \left(u_{0}(x)\right) d x<\infty
$$

where $u_{0}$ is the density of $\mu_{0}$. 


\section{PRELIMINARIES}

We begin by looking at a Poincaré inequality for our model. Let us remind the reader that the invariant probability $\mu^{\sigma, N}$ is of the form $e^{-U}$. According to [2], it satisfies a Poincaré inequality under simple assumptions. Indeed:

Proposition 2.1. Let $k$ be a positive integer. Let $\mu(d x):=e^{-U(x)} d x$ be a probability measure on $\mathbb{R}^{k}$. We assume that the potential $U$ is $\mathcal{C}^{2}$ and bounded from below. If there exist $\alpha>0$ and $R \geqslant 0$ such that, for $|x| \geqslant R$,

$$
\langle x ; \nabla U(x)\rangle \geqslant \alpha|x|,
$$

then $\mu$ satisfies a Poincaré inequality with constant

$$
\frac{4\left(1+\left(\exp \left\{\frac{1}{2}(\alpha R+1-k)\right\}+1\right) \kappa_{R}\right)}{(\alpha-(k-1) / R)^{2}}
$$

for any $R$ such that $\alpha-(k-1) / R>0$. Here, $\kappa_{R}$ is the Poincaré constant of $\mu$ restricted to the ball $\mathbb{B}(0 ; R)$.

The proof is omitted, see [2]. The general idea is the following. We can apply Theorem 1.4 in [2]. Indeed, we consider a sequence of smooth functions $W_{n}$ which satisfies

$$
W_{n}(x)= \begin{cases}\exp \left\{\frac{1}{2}(\alpha-(k-1) / R)|x|\right\} & \text { for }|x| \geqslant R, \\ \exp \left\{\frac{1}{2}\left(\alpha R+1-k-1 / n^{2}\right)\right\} & \text { for }|x| \leqslant R-1 / n .\end{cases}
$$

Consequently, we have the inequality

$$
\begin{aligned}
\Delta W_{n}(x)-\left\langle\nabla W_{n}(x) ; \nabla U(x)\right\rangle & \\
\leqslant & -\frac{1}{4}\left(\alpha-\frac{k-1}{R}\right)^{2} W_{n}(x) \\
& +\left(\exp \left\{\frac{1}{2}\left(\alpha R+1-k-\frac{1}{n^{2}}\right)\right\}+1\right) \mathbb{1}_{\mathbb{B}(0 ; R)}(x) .
\end{aligned}
$$

We may apply this proposition to our model under the assumptions (A1)-(A7).

We now give two classical results on functional inequalities.

PROPOSITION 2.2. Let $\mu$ be a probability measure on $\mathbb{R}^{k}$, and $U$ be a bounded function from $\mathbb{R}^{k}$ to $\mathbb{R}$. We define the measure $\nu$ - the perturbation of $\mu$ by $U$-as follows:

$$
d \nu:=\frac{e^{U}}{Z} d \mu \quad \text { with } Z:=\int e^{U} d \mu .
$$

If $\mu$ satisfies a Poincaré inequality with constant $C$, then $\nu$ satisfies a Poincaré inequality with constant $C \exp \left\{\sup _{\mathbb{R}^{k}} U-\inf _{\mathbb{R}^{k}} U\right\}$. 
Proof. Let $f$ be any smooth function. For $a=\int_{\mathbb{R}^{k}} f(y) d \mu(y)$ we have

$$
\begin{aligned}
\int_{\mathbb{R}^{k}}(f(x)-a)^{2} d \nu(x) & =\frac{1}{Z} \int_{\mathbb{R}^{k}}(f(x)-a)^{2} e^{U(x)} d \mu(x) \\
& \leqslant \frac{1}{Z} \exp \left\{\sup _{\mathbb{R}^{k}} U\right\} \int_{\mathbb{R}^{k}}(f(x)-a)^{2} d \mu(x) \\
& \leqslant \frac{C}{Z} \exp \left\{\sup _{\mathbb{R}^{k}} U\right\} \int_{\mathbb{R}^{k}}\|\nabla f(x)\|^{2} d \mu(x) \\
& \leqslant C \exp \left\{\sup _{\mathbb{R}^{k}} U\right\} \int_{\mathbb{R}^{k}}\|\nabla f(x)\|^{2} e^{-U(x)} d \nu(x) \\
& \leqslant C \exp \left\{\sup _{\mathbb{R}^{k}} U-\inf _{\mathbb{R}^{k}} U\right\} \int_{\mathbb{R}^{k}}\|\nabla f(x)\|^{2} d \nu(x) .
\end{aligned}
$$

As $\operatorname{Var}_{\nu}(f)$ is the infimum of $\int_{\mathbb{R}^{k}}(f(x)-a)^{2} d \nu(x)$ for $a \in \mathbb{R}^{k}$, this completes the proof.

Another well-known result is the tensorization one. We present it in $\mathbb{R}^{2}$ without any loss of generality.

PROPOSITION 2.3. Let $\mu_{1}$ and $\mu_{2}$ be two probability measures on $\mathbb{R}$. We assume that both probability measures $\mu_{1}$ and $\mu_{2}$ satisfy a Poincaré inequality with constant $C$. Then the probability measure on $\mathbb{R}^{2}, \mu_{1} \otimes \mu_{2}$, satisfies a Poincaré inequality with constant $C$.

Proof. For any smooth function from $\mathbb{R}^{2}$ to $\mathbb{R}$, one can easily prove the inequality

$$
\operatorname{Var}_{\mu_{1} \otimes \mu_{2}}(f) \leqslant \mathbb{E}_{\mu_{1} \otimes \mu_{2}}\left(\operatorname{Var}_{\mu_{1}}(f)\right)+\mathbb{E}_{\mu_{1} \otimes \mu_{2}}\left(\operatorname{Var}_{\mu_{2}}(f)\right),
$$

where $\operatorname{Var}_{\mu_{1}}$ (respectively, $\operatorname{Var}_{\mu_{2}}$ ) means that the first (respectively, the second) variable is the only one to be affected by the integration. Indeed, this inequality is equivalent to

$$
\mathbb{E}_{\mu_{1} \otimes \mu_{2}}\left(f^{2}\right)-\mathbb{E}_{\mu_{1} \otimes \mu_{2}}\left[\left(\mathbb{E}_{\mu_{1}}(f)\right)^{2}\right]-\mathbb{E}_{\mu_{1} \otimes \mu_{2}}\left[\left(\mathbb{E}_{\mu_{2}}(f)\right)^{2}\right]+\left(\mathbb{E}_{\mu_{1} \otimes \mu_{2}}(f)\right)^{2} \geqslant 0,
$$

which can also be written as

$$
\mathbb{E}_{\mu_{1} \otimes \mu_{2}}\left[\left(f-\mathbb{E}_{\mu_{1}}(f)-\mathbb{E}_{\mu_{2}}(f)+\mathbb{E}_{\mu_{1} \otimes \mu_{2}}(f)\right)^{2}\right] \geqslant 0 .
$$

However, the Poincaré inequality implies

$$
\operatorname{Var}_{\mu_{1}}(f) \leqslant C \int_{\mathbb{R}}\left|\nabla_{x_{1}} f\right|^{2} d \mu_{1} \quad \text { and } \quad \operatorname{Var}_{\mu_{2}}(f) \leqslant C \int_{\mathbb{R}}\left|\nabla_{x_{2}} f\right|^{2} d \mu_{2},
$$

which completes the proof. 
We proceed in a similar way for the tensorization of $k$ measures on $\mathbb{R}$.

In the model considered we have a Poincaré inequality. However, the constant may depend on the dimension.

The following constant, which appears in Proposition 2.1], does depend on the dimension:

$$
\frac{4\left(1+\left(\exp \left\{\frac{1}{2}(\alpha R+1-k)\right\}+1\right) \kappa_{R}\right)}{(\alpha-(k-1) / R)^{2}} .
$$

Indeed, $R$ has to be such that $\alpha>(k-1) / R$, which means that $R>(k-1) / \alpha$. Consequently, the constant is greater than

$$
\frac{4\left(1+2 \kappa_{(k-1) / R}\right)}{(\alpha-(k-1) / R)^{2}} .
$$

However, we can remark that $\lim _{R \rightarrow \infty} \kappa_{R}=+\infty$.

By using the tensorization result, we easily prove that the measure

$$
\exp \left\{-\frac{2}{\sigma^{2}} \sum_{k=1}^{N} V\left(x_{k}\right)\right\} d x_{1} \ldots d x_{N}
$$

satisfies a Poincaré inequality with a constant which does not depend on the dimension $N$. If we assume that $F$ is bounded, we can use the perturbation result to prove that the measure $\exp \left\{-\frac{2}{\sigma^{2}} N \Upsilon_{N}\left(x_{1}, \ldots, x_{N}\right)\right\} d x_{1} \ldots d x_{N}$ satisfies a Poincaré inequality. However, the constant just obtained does depend on the dimension.

Let us observe that we can write

$$
N \Upsilon^{N}\left(x_{1}, \ldots, x_{N}\right)=\sum_{k=1}^{N}\left(V\left(x_{k}\right)+F * \eta^{\mathcal{X}}\left(x_{k}\right)\right),
$$

with $\eta^{\mathcal{X}}:=\frac{1}{N} \sum_{k=1}^{N} \delta_{x_{k}}$. However, we cannot use the tensorization result. Intuitively, the propagation of chaos means that the particles become independent so that we have a Poincaré inequality with a constant which does not depend on the dimension.

In the following, we deal with $W J$-inequality to get inequality independent of the dimension.

\section{FRAMEWORK}

Let us give the framework (definitions and basic propositions) of our paper. We begin by introducing the Wasserstein distance.

DEFINITION 3.1. For any probability measures on $\mathbb{R}^{d}, \mu$ and $\nu$, the Wasserstein distance between $\mu$ and $\nu$ is

$$
\mathbb{W}_{2}(\mu ; \nu):=\sqrt{\inf \mathbb{E}\left\{\|X-Y\|^{2}\right\}},
$$


where the infimum is taken over the random variables $X$ and $Y$ with law $\mu$ and $\nu$, respectively.

By Brenier's theorem, see [ 7$]$, the Wasserstein distance can be characterized in the following way.

Proposition 3.1. Let $\mu$ and $\nu$ be two probability measures on $\mathbb{R}^{d}$. If $\mu$ is absolutely continuous with respect to the Lebesgue measure, there exists a convex function $\tau$ from $\mathbb{R}^{d}$ to $\mathbb{R}$ such that the following equality holds for every bounded test function $g$ :

$$
\int_{\mathbb{R}^{d}} g(x) \nu(d x)=\int_{\mathbb{R}^{d}} g(\nabla \tau(x)) \mu(d x) .
$$

Then, we write

$$
\nu=\nabla \tau \# \mu,
$$

and we have the following equality:

$$
\mathbb{W}_{2}(\mu ; \nu)=\sqrt{\int_{\mathbb{R}^{d}}\|x-\nabla \tau(x)\|^{2} \mu(d x)} .
$$

The key idea of the paper is a so-called $W J_{V, F}$-inequality. Let us present the expression that we denote by $J_{V, F}(\nu \mid \mu)$ if $\mu$ is absolutely continuous with respect to the Lebesgue measure:

$$
\begin{aligned}
& J_{V, F}(\nu \mid \mu):=J_{V, 0}(\nu \mid \mu) \\
& \quad+\frac{1}{2} \iint_{\mathbb{R}^{2 d}}\langle\nabla F(\xi(x, y))-\nabla F(x-y) ; \xi(x, y)-(x-y)\rangle \mu(d x) \mu(d y),
\end{aligned}
$$

with $\xi(x, y):=\nabla \tau(x)-\nabla \tau(y)$ and

$$
\begin{aligned}
J_{V, 0}(\nu \mid \mu):= & \frac{\sigma^{2}}{2} \int_{\mathbb{R}^{d}}\left(\Delta \tau(x)+\Delta \tau^{*}(\nabla \tau(x))-2 d\right) \mu(d x) \\
& +\int_{\mathbb{R}^{d}}\langle\nabla V(\nabla \tau(x))-\nabla V(x) ; \nabla \tau(x)-x\rangle \mu(d x),
\end{aligned}
$$

where $\tau^{*}$ denotes the Legendre transform of $\tau$. Here, we have $\nu=\nabla \tau \# \mu$. We now present the transportation inequality, already used in [1], [5], [6], [13], on which the article is based.

DEFINITION 3.2. Let $\mu$ be a probability measure on $\mathbb{R}^{d}$ absolutely continuous with respect to the Lebesgue measure and $C>0$. We say that $\mu$ satisfies a $W J_{V, F}(C)$-inequality if the inequality

$$
C \mathbb{W}_{2}^{2}(\nu ; \mu) \leqslant J_{V, F}(\nu \mid \mu)
$$

holds for any probability measure $\nu$ on $\mathbb{R}^{d}$. 
In the following, we aim to establish $W J$-inequality for the invariant probability $\mu^{\sigma, N}$ of diffusion (ㅍ. I). It is well known that $\mu^{\sigma, N}$ is absolutely continuous with respect to the Lebesgue measure. Consequently, we can apply Brenier's theorem. So, the $W J_{V, F}$-inequality reduces to an inequality on the convex functions $\tau$ from $\mathbb{R}^{d}$ to $\mathbb{R}$.

Let us remark that a $W J_{V, F}$-inequality does imply the required convergence. Indeed, it is a consequence of Proposition 1.1 in [6].

\section{MAIN RESULTS}

Here we use the result from [5]. We know that a $W J_{V, 0}$-inequality holds with some constant $C_{\sigma, N}$. Therefore, a $W J_{V, F}$-inequality holds with the constant $C_{\sigma, N}-(\alpha+\theta)$ :

PROPOSITION 4.1. Under the assumptions (A1)-(A7), a $W J_{V, F}$-inequality holds for the measure $\mu^{\sigma, N}$ with the constant $C_{\sigma, N}-(\alpha+\theta)$, where

$$
C_{\sigma, N}:=\max _{R>0} C_{\sigma}(N, R),
$$

and

$$
\begin{aligned}
C_{\sigma}(N, R):=\min \left\{\frac{K(R)}{3} ; \frac{\sigma^{2}}{72 R^{2}} \exp \left\{-\frac{2}{\sigma^{2}} S(R)\right\}\right. \\
\left.\frac{K(R)}{6} \frac{3^{d N}-2^{d N}}{2^{d N}} \exp \left\{\frac{2}{\sigma^{2}}(I(R)-S(R))\right\}\right\},
\end{aligned}
$$

with

$$
\begin{aligned}
K(R) & :=\inf _{\|\chi\| \geqslant R} N \nabla^{2} \Upsilon_{0}^{N}(\chi), \\
I(R) & :=\inf _{\|\chi\| \leqslant 2 R} N \Upsilon^{N}(\chi), \\
S(R) & :=\sup _{\|\chi\| \leqslant 3 R} N \Upsilon^{N}(\chi) .
\end{aligned}
$$

In the definition of $K(R)$, the infimum is understood as the smallest eigenvalue of $N \nabla^{2} \Upsilon_{0}^{N}(\chi)$. Furthermore, $\Upsilon_{0}^{N}$ is defined as follows:

$$
N \Upsilon_{0}^{N}(\chi):=\sum_{i=1}^{N} V_{0}\left(\chi_{i}\right)+\frac{1}{2 N} \sum_{i=1}^{N} \sum_{j=1}^{N} G\left(\left\|\chi_{i}-\chi_{j}\right\|\right) .
$$

This proposition is Proposition 3.4 of [6] with explicit constants. For reader's convenience, in Section 5 we repeat the proof from [6], tracking the constants carefully.

However, nothing ensures us a priori that $C_{\sigma, N}-(\alpha+\theta)$ is positive. This is the aim of the next theorem: 
THEOREM 4.1. There exists $\widehat{\sigma}_{c}$ such that $C_{\sigma, N}>\alpha+\theta$ for any $N$ if $\sigma \geqslant \widehat{\sigma}_{c}$.

Pro of. We will use Proposition 4.1] and the notation introduced therein. From (A2) and (A3) we get

$$
\nabla^{2} N \Upsilon_{0}^{N}(\chi) \geqslant(12 \alpha+12 \theta+12) \text { Id } \quad \text { if }\|\chi\| \geqslant R:=R_{12 \alpha+11 \theta+12} .
$$

Therefore,

$$
\frac{K(R)}{3} \geqslant 4(\alpha+\theta+1)
$$

From (A4) and (A5) it follows that

$$
|V(x)| \leqslant C\left(\|x\|+\frac{\|x\|^{2 m}}{2 m}\right), \quad x \in \mathbb{R}^{d},
$$

which together with (A5) implies that

$$
|V(x)| \leqslant c\left(\|x\|^{2}+\|x\|^{2 m}\right), \quad x \in \mathbb{R}^{d} .
$$

On the other hand, from (A6) we get $F(x)=G(\|x\|)-\frac{\alpha}{2}\|x\|^{2}=\sum_{k=1}^{n} b_{2 k}\|x\|^{2 k}$ for some $b_{2 k} \in \mathbb{R}$. This and (4.6) give

$$
\begin{aligned}
N \Upsilon^{N}(\chi) & =\sum_{i=1}^{N} V\left(\chi_{i}\right)+\frac{1}{2 N} \sum_{i=1}^{N} \sum_{j=1}^{N} F\left(\chi_{i}-\chi_{j}\right) \\
& \leqslant c \sum_{i=1}^{N}\left(\left\|\chi_{i}\right\|^{2}+\left\|\chi_{i}\right\|^{2 m}\right)+\frac{1}{2 N} \sum_{i=1}^{N} \sum_{j=1}^{N} \sum_{k=1}^{n} b_{2 k}\left\|\chi_{i}-\chi_{j}\right\|^{2 k} .
\end{aligned}
$$

We continue, using the elementary inequality $|a-b|^{2 k} \leqslant 2^{2 k-1}\left(|a|^{2 k}+|b|^{2 k}\right)$, and obtain

$$
\begin{aligned}
N \Upsilon^{N}(\chi) & \leqslant c \sum_{i=1}^{N}\left(\left\|\chi_{i}\right\|^{2}+\left\|\chi_{i}\right\|^{2 m}\right)+\sum_{k=1}^{n} b_{2 k} 2^{2 k-1} \sum_{i=1}^{N}\left\|\chi_{i}\right\|^{2 k} \\
& \leqslant c\left(9 R^{2}+9^{m} R^{2 m}\right)+\sum_{k=1}^{n} b_{2 k} 2^{2 k-1} 9^{k} R^{2 k} \leqslant c^{\prime} R^{2 q} \quad \text { if }\|\chi\| \leqslant 3 R,
\end{aligned}
$$

where $q=\max \{m, n\}$ and the constant $c^{\prime}$ depends only on $V$ and $F$. Thus,

$$
S(R) \leqslant c^{\prime} R^{2 q} .
$$

Finally, to estimate $I(R)$, we use (A3) and (A6). For $\|\chi\| \leqslant 2 R$,

$$
\begin{aligned}
N \Upsilon^{N}(\chi) & \geqslant-\frac{\theta}{2} \sum_{i=1}^{N}\left\|\chi_{i}\right\|^{2}-\frac{\alpha}{4 N} \sum_{i=1}^{N} \sum_{j=1}^{N}\left\|\chi_{i}-\chi_{j}\right\|^{2} \\
& \geqslant-2 \theta R^{2}-\frac{\alpha}{4 N} \sum_{i=1}^{N} \sum_{j=1}^{N} 2\left(\left\|\chi_{i}\right\|^{2}+\left\|\chi_{j}\right\|^{2}\right) \geqslant-(2 \theta+4 \alpha) R^{2},
\end{aligned}
$$


hence

$$
I(R) \geqslant-(2 \theta+4 \alpha) R^{2} .
$$

We are now ready to estimate $C_{\sigma}(N, R)$. The first term in the minimum on the right-hand side of (4. 1 ) is greater than $4(\alpha+\theta+1)$, see (4.5)). The second term is greater than

$$
\frac{\sigma^{2}}{72 R^{2}} \exp \left\{-\frac{2}{\sigma^{2}} c^{\prime} R^{2 q}\right\}
$$

see (4.8), which in turn is larger than $\alpha+\theta+1$ for $\sigma$ large enough. Finally, the third term in the minimum in (4.1) is greater than

$$
2(\alpha+\theta+1) \frac{1}{2} \exp \left\{\frac{2}{\sigma^{2}}\left(-(2 \theta+4 \alpha) R^{2}-c^{\prime} R^{2 q}\right)\right\} \geqslant \alpha+\theta+\frac{1}{2}
$$

for large $\sigma$. To summarize, $C_{\sigma}(N, R) \geqslant \alpha+\theta+\frac{1}{2}$ and, consequently,

$$
C_{\sigma, N} \geqslant \alpha+\theta+\frac{1}{2}
$$

for every $N \in \mathbb{N}$ and for large $\sigma$.

Pro of of The or e $\mathrm{m}$ ㄸ.]. From Theorem 4.], if the diffusion coefficient $\sigma$ is large enough, a $W J_{V, F}$-inequality holds with a constant which does not depend on the dimension. Thus the assertion follows from Proposition 1.1 in [6].

\section{PROOF OF PROPOSITION 4.1}

Here, we consider a monotone map $A$ on $\mathbb{R}^{n}$, and by $\nabla^{S} A:=\frac{\nabla A+\nabla A^{T}}{2}$ we denote the symmetric part of its Jacobian.

We remind Lemma 3.12 of [5]:

Lemma 5.1. Let $A$ be a $\mathcal{C}^{1}$ monotone map on $\mathbb{R}^{n}$ for which there exist two constants $R$ and $K>0$ such that $\nabla^{S} A(x) \geqslant K$ for all $|x| \geqslant R$. Then

$$
\langle A(x)-A(y) ; x-y\rangle \geqslant \frac{K}{3}|x-y|^{2}
$$

if $|x| \geqslant 2 R$ or $|y| \geqslant 2 R$.

Pro of of Proposition 4. W. We follow carefully the proof in Section 3.5 of [5]. Next pages are an adaptation with $\sigma \neq 1$ of Section 3.5 in [5].

Let $\varphi$ be a given strictly convex function on $\mathbb{R}^{n}$. Let us recall that, for the Hessian operator,

$$
\nabla^{2} \varphi^{*}(\nabla \varphi(x))=\left(\nabla^{2} \varphi(x)\right)^{-1}
$$


and, in particular,

$$
\Delta \varphi^{*}(\nabla \varphi(x))=\operatorname{Tr}\left[\left(\nabla^{2} \varphi(x)\right)^{-1}\right]
$$

Tr being the trace operator.

Let $X$ be the subset of $\mathbb{R}^{n}$ defined by

$$
X:=\left\{x \in \mathbb{R}^{n}:|x| \leqslant 2 R,|\nabla \varphi(x)| \leqslant 2 R\right\} .
$$

S te $\mathrm{p}$ 1. First of all, by monotonicity and Lemma [.], we have

$$
\begin{aligned}
\int_{\mathbb{R}^{n}}\langle A(\nabla \varphi(x))-A(x) ; \nabla \varphi(x)-x\rangle \exp \left\{-\frac{2}{\sigma^{2}} V(x)\right\} d x \\
\geqslant \int_{X^{c}}\langle A(\nabla \varphi(x))-A(x) ; \nabla \varphi(x)-x\rangle \exp \left\{-\frac{2}{\sigma^{2}} V(x)\right\} d x \\
\geqslant \frac{K}{3} \int_{X^{c}}|\nabla \varphi(x)-x|^{2} \exp \left\{-\frac{2}{\sigma^{2}} V(x)\right\} d x .
\end{aligned}
$$

S te $\mathrm{p} 2$. On the other hand, for $\theta \in \mathbb{S}^{n-1}$ we let $R_{\theta}:=\sup \{r \geqslant 0: r \theta \in X\}$. In particular, $R_{\theta} \theta \in X$ and $R_{\theta} \leqslant 2 R$. Then, we let $r_{\theta} \in\left[R_{\theta}, 3 R\right]$ such that

$$
\left|\nabla \varphi\left(r_{\theta} \theta\right)-r_{\theta} \theta\right|=\inf \left\{|\nabla \varphi(r \theta)-r \theta|: r \in\left[R_{\theta}, 3 R\right]\right\} .
$$

In particular,

$$
\begin{aligned}
\left|\nabla \varphi\left(r_{\theta} \theta\right)\right| & \leqslant\left|\nabla \varphi\left(r_{\theta} \theta\right)-r_{\theta} \theta\right|+\left|r_{\theta} \theta\right| \\
& \leqslant\left|\nabla \varphi\left(R_{\theta} \theta\right)-R_{\theta} \theta\right|+\left|r_{\theta} \theta\right| \\
& \leqslant 2 R+2 R+3 R=7 R,
\end{aligned}
$$

since $\left|\nabla \varphi\left(R_{\theta} \theta\right)\right| \leqslant 2 R$ and $\left|R_{\theta} \theta\right| \leqslant 2 R$ for $R_{\theta} \theta \in X$.

Then, for $r \theta \in X$ with $0 \leqslant r \leqslant R_{\theta} \leqslant r_{\theta}$, let us write

$$
\nabla \varphi(r \theta)-r \theta=\nabla \varphi\left(r_{\theta} \theta\right)-r_{\theta} \theta+\int_{r_{\theta}}^{r}\left[\nabla^{2} \varphi(s \theta)-I\right] \theta d s .
$$

Letting $H_{s}:=\nabla^{2} \varphi(s \theta)$ for notational convenience, we decompose as

$$
\left[H_{s}-I\right] \theta=\left[H_{s}^{1 / 2}-H_{s}^{-1 / 2}\right] H_{s}^{1 / 2} \theta,
$$

so that

$$
\begin{aligned}
\left|\int_{r}^{r_{\theta}}\left[H_{s}-I\right] \theta d s\right|^{2} & \leqslant\left(\int_{r}^{r_{\theta}}\left|H_{s}^{1 / 2}-H_{s}^{-1 / 2}\right|\left|H_{s}^{1 / 2} \theta\right| d s\right)^{2} \\
\leqslant & \left(\int_{r}^{r_{\theta}}\left|H_{s}^{1 / 2}-H_{s}^{-1 / 2}\right|^{2} \exp \left\{-\frac{2}{\sigma^{2}} V(s \theta)\right\} d s\right) \\
& \times\left(\int_{r}^{r_{\theta}}\left|H_{s}^{1 / 2} \theta\right|^{2} \exp \left\{\frac{2}{\sigma^{2}} V(s \theta)\right\} d s\right)
\end{aligned}
$$


by the Hölder inequality. But

$$
\begin{aligned}
\left|H_{s}^{1 / 2}-H_{s}^{-1 / 2}\right|^{2} & =\sup _{x \in \mathbb{R}^{n}} \frac{\left|\left[H_{s}^{1 / 2}-H_{s}^{-1 / 2}\right] x\right|^{2}}{|x|^{2}}=\sup _{x \in \mathbb{R}^{n}} \frac{\left\langle\left[H_{s}-2 I+H_{s}^{-1}\right] x ; x\right\rangle}{|x|^{2}} \\
& \leqslant \operatorname{Tr}\left(H_{s}-2 I+H_{s}^{-1}\right)=\Delta \varphi(s \theta)-2 n+\left(\Delta \varphi^{*}\right)(\nabla \varphi(s \theta)),
\end{aligned}
$$

since the eigenvalues of $H-2 I+H^{-1}$ are non-negative. Moreover,

$$
\left|H_{s}^{1 / 2} \theta\right|^{2}=\left\langle H_{s}^{1 / 2} \theta ; H_{s}^{1 / 2} \theta\right\rangle=\left\langle H_{s} \theta ; \theta\right\rangle .
$$

Hence,

$$
\begin{aligned}
|\nabla \varphi(r \theta)-r \theta|^{2} \leqslant & 2\left|\nabla \varphi\left(r_{\theta} \theta\right)-r_{\theta} \theta\right|^{2} \\
& +2\left(\int_{r}^{r_{\theta}}\left(\Delta \varphi(s \theta)-2 n+\Delta \varphi^{*}(\nabla \varphi(s \theta))\right) \exp \left\{-\frac{2}{\sigma^{2}} V(s \theta)\right\} d s\right) \\
& \times\left(\int_{r}^{r_{\theta}}\left\langle H_{s} \theta ; \theta\right\rangle \exp \left\{\frac{2}{\sigma^{2}} V(s \theta)\right\} d s\right),
\end{aligned}
$$

where

$$
\int_{r}^{r_{\theta}}\left\langle H_{s} \theta ; \theta\right\rangle d s=\left\langle\nabla \varphi\left(r_{\theta} \theta\right)-\nabla \varphi(r \theta) ; \theta\right\rangle \leqslant\left|\nabla \varphi\left(r_{\theta} \theta\right)\right|+|\nabla \varphi(r \theta)| \leqslant 9 R
$$

for $r \theta \in X$. Hence,

$$
\begin{aligned}
\int_{X,|x| \leqslant 2 R}|\nabla \varphi(x)-x|^{2} \exp \left\{-\frac{2}{\sigma^{2}} V(x)\right\} d x \\
\leqslant \int_{\mathbb{S}^{n-1}} \int_{0}^{R_{\theta}} r^{n-1}|\nabla \varphi(r \theta)-r \theta|^{2} \exp \left\{-\frac{2}{\sigma^{2}} V(r \theta)\right\} d r d \theta \\
\leqslant 2 \int_{\mathbb{S}^{n-1}} \int_{0}^{R_{\theta}}\left|\nabla \varphi\left(r_{\theta} \theta\right)-r_{\theta} \theta\right|^{2} \exp \left\{-\frac{2}{\sigma^{2}} V(r \theta)\right\} d r d \theta \\
\quad+\lambda(R) \int_{\mathbb{S}^{n-1}} \int_{0}^{R_{\theta}} r^{n-1} \int_{r}^{r_{\theta}}\left(\Delta \varphi(s \theta)-2 n+\Delta \varphi^{*}(\nabla \varphi(s \theta))\right) \\
\quad \times \exp \left\{-\frac{2}{\sigma^{2}} V(s \theta)\right\} d s d r d \theta
\end{aligned}
$$

where

$$
\lambda(R):=18 R \exp \left\{\frac{2}{\sigma^{2}} \sup \{V(x):|x| \leqslant 3 R\}\right\} .
$$


But,

$$
\begin{aligned}
& \int_{\mathbb{S}^{n-1}} \int_{0}^{R_{\theta}} r^{n-1} \int_{r}^{r_{\theta}}\left(\Delta \varphi(s \theta)-2 n+\Delta \varphi^{*}(\nabla \varphi(s \theta))\right) \exp \left\{-\frac{2}{\sigma^{2}} V(s \theta)\right\} d s d r d \theta \\
\leqslant & \int_{\mathbb{S}^{n-1}} \int_{0}^{R_{\theta}} \int_{r}^{r_{\theta}} s^{n-1}\left(\Delta \varphi(s \theta)-2 n+\Delta \varphi^{*}(\nabla \varphi(s \theta))\right) \exp \left\{-\frac{2}{\sigma^{2}} V(s \theta)\right\} d s d r d \theta \\
\leqslant & 2 R \int_{\mathbb{S}^{n-1}} \int_{0}^{3 R} s^{n-1}\left(\Delta \varphi(s \theta)-2 n+\Delta \varphi^{*}(\nabla \varphi(s \theta))\right) \exp \left\{-\frac{2}{\sigma^{2}} V(s \theta)\right\} d s d \theta \\
= & \frac{4 R}{\sigma^{2}} \cdot \frac{\sigma^{2}}{2} \int_{|x| \leqslant 3 R}\left(\Delta \varphi(x)-2 n+\Delta \varphi^{*}(\nabla \varphi(x))\right) \exp \left\{-\frac{2}{\sigma^{2}} V(x)\right\} d x .
\end{aligned}
$$

Hence,

$$
\begin{aligned}
& \text { (5.1) } \int_{X,|x| \leqslant 2 R}|\nabla \varphi(x)-x|^{2} \exp \left\{-\frac{2}{\sigma^{2}} V(x)\right\} d x \\
& \leqslant 2 \exp \left\{-\frac{2}{\sigma^{2}} \inf \{V(x):|x| \leqslant 2 R\}\right\} \frac{(2 R)^{n}}{n} \int_{\mathbb{S}^{n-1}}\left|\nabla \varphi\left(r_{\theta} \theta\right)-r_{\theta} \theta\right|^{2} d \theta \\
& +\frac{4 R}{\sigma^{2}} \lambda(R) \cdot \frac{\sigma^{2}}{2} \int_{|x| \leqslant 3 R}\left(\Delta \varphi(x)-2 n+\Delta \varphi^{*}(\nabla \varphi(x))\right) \exp \left\{-\frac{2}{\sigma^{2}} V(x)\right\} d x .
\end{aligned}
$$

Moreover, by Lemma 5.$]$ and the definition of $r_{\theta}$, we obtain

$$
\begin{aligned}
& \int_{|x| \leqslant 3 R}\langle A(\nabla \varphi(x))-A(x) ; \nabla \varphi(x)-x\rangle \exp \left\{-\frac{2}{\sigma^{2}} V(x)\right\} d x \\
\geqslant & \int_{2 R \leqslant|x| \leqslant 3 R}\langle A(\nabla \varphi(x))-A(x) ; \nabla \varphi(x)-x\rangle \exp \left\{-\frac{2}{\sigma^{2}} V(x)\right\} d x \\
\geqslant & \frac{K}{3} \int_{2 R \leqslant|x| \leqslant 3 R}|\nabla \varphi(x)-x|^{2} \exp \left\{-\frac{2}{\sigma^{2}} V(x)\right\} d x \\
\geqslant & \frac{K}{3} \exp \left\{-\frac{2}{\sigma^{2}} \sup \{V(x):|x| \leqslant 3 R\}\right\} \int_{2 R \leqslant|x| \leqslant 3 R}|\nabla \varphi(x)-x|^{2} d x \\
= & \frac{K}{3} \exp \left\{-\frac{2}{\sigma^{2}} \sup \{V(x):|x| \leqslant 3 R\}\right\} \int_{2 R}^{3 R} r^{n-1} \int_{\mathbb{S}^{n-1}}|\nabla \varphi(r \theta)-r \theta|^{2} d \theta d r \\
\geqslant & \frac{K}{3} \exp \left\{-\frac{2}{\sigma^{2}} \sup \{V(x):|x| \leqslant 3 R\} \int_{2 R}^{3 R} r^{n-1} \int_{\mathbb{S}^{n-1}}\left|\nabla \varphi\left(r_{\theta} \theta\right)-r_{\theta} \theta\right|^{2} d \theta d r .\right.
\end{aligned}
$$


Hence, there exists a constant $C(R, K)$ such that

$$
\begin{aligned}
& C(R, K) \int_{\mathbb{S}^{n-1}}\left|\nabla \varphi\left(r_{\theta} \theta\right)-r_{\theta} \theta\right|^{2} d \theta \\
& \quad \leqslant \int_{|x| \leqslant 3 R}\langle A(\nabla \varphi(x))-A(x) ; \nabla \varphi(x)-x\rangle \exp \left\{-\frac{2}{\sigma^{2}} V(x)\right\} d x .
\end{aligned}
$$

Thus, we have

$$
C(R, K):=\frac{K}{3} \exp \left\{-\frac{2}{\sigma^{2}} \sup \{V(x):|x| \leqslant 3 R\}\right\} \frac{1}{n}\left(3^{n}-2^{n}\right) R^{n} .
$$

It follows from (5.11) and (5.2) that

$$
\begin{aligned}
& C^{\prime}(R, K) \int_{X,|x| \leqslant 2 R}|\nabla \varphi(x)-x|^{2} \exp \left\{-\frac{2}{\sigma^{2}} V(x)\right\} d x \\
& \leqslant \int_{|x| \leqslant 3 R}\langle A(\nabla \varphi(x))-A(x) ; \nabla \varphi(x)-x\rangle \exp \left\{-\frac{2}{\sigma^{2}} V(x)\right\} d x \\
& \quad+\frac{\sigma^{2}}{2} \int_{|x| \leqslant 3 R}\left(\Delta \varphi(x)-2 n+\Delta \varphi^{*}(\nabla \varphi(x))\right) \exp \left\{-\frac{2}{\sigma^{2}} V(x)\right\} d x,
\end{aligned}
$$

where

$$
\begin{aligned}
C^{\prime}(R, K):=\min \left\{\frac{\sigma^{2}}{72 R^{2}} \exp \{\right. & \left.-\frac{2}{\sigma^{2}} S(R)\right\} ; \\
& \left.\frac{K}{6} \frac{3^{n}-2^{n}}{2^{n}} \exp \left\{\frac{2}{\sigma^{2}}(I(R)-S(R))\right\}\right\},
\end{aligned}
$$

with $I(R):=\inf _{|x| \leqslant 2 R} V$ and $S(R):=\sup _{|x| \leqslant 3 R} V$.

Moreover,

$$
\begin{aligned}
\int_{|x| \leqslant 3 R}\langle A(\nabla \varphi(x)) & -A(x) ; \nabla \varphi(x)-x\rangle \exp \left\{-\frac{2}{\sigma^{2}} V(x)\right\} d x \\
& \geqslant \frac{K}{3} \int_{2 R \leqslant|x| \leqslant 3 R}|\nabla \varphi(x)-x|^{2} \exp \left\{-\frac{2}{\sigma^{2}} V(x)\right\} d x,
\end{aligned}
$$

so that

$$
\begin{aligned}
& C^{\prime \prime}(R, K) \int_{X}|\nabla \varphi(x)-x|^{2} \exp \left\{-\frac{2}{\sigma^{2}} V(x)\right\} d x \\
& \leqslant \int_{|x| \leqslant 3 R}\langle A(\nabla \varphi(x))-A(x) ; \nabla \varphi(x)-x\rangle \exp \left\{-\frac{2}{\sigma^{2}} V(x)\right\} d x \\
& \quad+\frac{\sigma^{2}}{2} \int_{|x| \leqslant 3 R}\left(\Delta \varphi(x)-2 n+\Delta \varphi^{*}(\nabla \varphi(x))\right) \exp \left\{-\frac{2}{\sigma^{2}} V(x)\right\} d x,
\end{aligned}
$$


where

$$
\begin{aligned}
& C^{\prime \prime}(R, K):=\min \left\{\frac{K}{3} ; \frac{\sigma^{2}}{72 R^{2}} \exp \left\{-\frac{2}{\sigma^{2}} S(R)\right\}\right. \\
&\left.\frac{K}{6} \frac{3^{n}-2^{n}}{2^{n}} \exp \left\{\frac{2}{\sigma^{2}}(I(R)-S(R))\right\}\right\} .
\end{aligned}
$$

Finally, the last two integrands are non-negative maps, so we can bound from above these last two integrals on the set $\{|x| \leqslant 3 R\}$ by the corresponding integrals on the whole $\mathbb{R}^{n}$.

S te p 3. We conclude the proof by adding the estimates in Steps 1 and 2. Then, we replace $V$ by $N \Upsilon^{N}$.

Acknowledgments. (B.D.): Part of the research was done while I was visiting Université Jean Monnet Saint-Étienne, and I would like to thank Julian Tugaut for his hospitality.

(J.T.): Velika hvala Marini za sve. Également, un très grand merci à Manue, à Sandra et à Virginie pour tout.

(B.D. and J.T.): We would like to thank the anonymous referee for careful reading and useful suggestions.

\section{REFERENCES}

[1] L. Ambrosio, N. Gigli, and G. Savaré, Gradient Flows in Metric Spaces and in the Space of Probability Measures, Birkhäuser, Basel 2008.

[2] D. Bakry, F. Barthe, P. Cattiaux, and A. Guillin, A simple proof of the Poincaré inequality for a large class of probability measures including the log-concave case, Electron. Commun. Probab. 13 (2008), pp. 60-66.

[3] S. Benachour, B. Roynette, D. Talay, and P. Vallois, Nonlinear self-stabilizing processes. I. Existence, invariant probability, propagation of chaos, Stochastic Process. Appl. 75 (2) (1998), pp. 173-201.

[4] G. Ben Arous and O. Zeitouni, Increasing propagation of chaos for mean field models, Ann. Inst. Henri Poincaré Probab. Stat. 35 (1) (1999), pp. 85-102.

[5] F. Bolley, I. Gentil, and A. Guillin, Convergence to equilibrium in Wasserstein distance for Fokker-Planck equations, J. Funct. Anal. 263 (8) (2012), pp. 2430-2457.

[6] F. Bolley, I. Gentil, and A. Guillin, Uniform convergence to equilibrium for granular media, Arch. Ration. Mech. Anal. 208 (2) (2013), pp. 429-445.

[7] Y. Brenier, Polar factorization and monotone rearrangement of vector-valued functions, Comm. Pure Appl. Math. 44 (1991), pp. 375-417.

[8] F. Collet, P. Dai Pra, and E. Sartori, A simple mean field model for social interactions: dynamics, fluctuations, criticality, J. Stat. Phys. 139 (5) (2010), pp. 820-858.

[9] D. Crisan and J. Xiong, Approximate McKean-Vlasov representations for a class of SPDEs, Stochastics 82 (1-3) (2010), pp. 53-68.

[10] H. P. McKean, Jr., A class of Markov processes associated with nonlinear parabolic equations, Proc. Natl. Acad. Sci. USA 56 (1966), pp. 1907-1911.

[11] H. P. McKean, Jr., Propagation of chaos for a class of non-linear parabolic equations, in: Stochastic Differential Equations (Lecture Series in Differential Equations, Session 7, Catholic Univ., 1967), Air Force Office Sci. Res., Arlington, VA, 1967, pp. 41-57. 
[12] S. Méléard, Asymptotic behaviour of some interacting particle systems; McKean-Vlasov and Boltzmann models, in: Probabilistic Models for Nonlinear Partial Differential Equations (Montecatini Terme, 1995), Lecture Notes in Math., Vol. 1627, Springer, Berlin 1996, pp. 42-95.

[13] K.-T. Sturm and M.-K. von Renesse, Transport inequalities, gradient estimates, entropy and Ricci curvature, Comm. Pure Appl. Math. 68 (2005), pp. 923-940.

[14] A-S. Sznitman, Topics in propagation of chaos, in: École d'Été de Probabilités de SaintFlour XIX-1989, Lecture Notes in Math., Vol. 1464, Springer, Berlin 1991, pp. 165-251.

Bartłomiej Dyda

Faculty of Pure and Applied Mathematics

Wrocław University of Science and Technology

Wybrzeże Wyspiańskiego 27

50-370 Wrocław, Poland

E-mail: bdyda@pwr.edu.pl
Julian Tugaut

Université Jean Monnet, Saint-Étienne and Institut Camille Jordan, Lyon 23, rue du docteur Paul Michelon, CS 82301 42023 Saint-Étienne Cedex 2, France E-mail: tugaut@math.cnrs.fr

Received on 19.5.2015,

revised version on 13.5.2016 